\title{
ILCEA
}

Revue de l'Institut des langues et cultures

d'Europe, Amérique, Afrique, Asie et Australie

36 | 2019

Représentations de la révolution de 1917 en Russie

contemporaine

\section{Un jeu des possibles en 1917 ?}

A Set of Possibilities in 1917?

\section{Tamara Kondratieva}

\section{(2) OpenEdition}

Journals

Édition électronique

URL : http://journals.openedition.org/ilcea/7326

DOI : 10.4000/ilcea.7326

ISSN : 2101-0609

\section{Éditeur}

UGA Éditions/Université Grenoble Alpes

\section{Édition imprimée}

ISBN : 978-2-37747-096-9

ISSN : 1639-6073

\section{Référence électronique}

Tamara Kondratieva, « Un jeu des possibles en 1917 ? », ILCEA [En ligne], 36 | 2019, mis en ligne le 21 juin 2019, consulté le 22 juin 2019. URL : http://journals.openedition.org/ilcea/7326 ; DOI : 10.4000/ ilcea.7326

Ce document a été généré automatiquement le 22 juin 2019.

(C) ILCEA 


\title{
Un jeu des possibles en $1917 ?$
}

\author{
A Set of Possibilities in 1917?
}

Tamara Kondratieva

\section{Introduction}

1 Partant de la glasnost' deux histoires d'Octobre s'écrivent en concurrence. Des publicistes et des amateurs s'adonnent rapidement et frénétiquement à des révélations sur l'exécution de la famille du tsar, "l'or allemand», la monstruosité de Lénine-espion allemand, la terreur rouge, la trahison des ouvriers et des paysans, etc. Suite à la levée de tous les précédents tabous, la révolution d'Octobre est alors délégitimée et ses héros discrédités. Ces usages publics de l'histoire transforment les mythes soviétiques positifs en mythes postsoviétiques négatifs, paraphrasant dans la plupart des cas l'historiographie de l'émigration russe des années 1920-1930. Un flot d'ouvrages auparavant interdits favorise un détour des « révisionnistes » et une tendance, parmi les historiens russes, à faire rebondir la conception libérale et totalitaire occidentale.

2 Dans le même temps, mais avec un moindre écho, une révision de l'histoire idéologisée d'Octobre déjà entamée en Occident par les « révisionnistes » perce en URSS sous le signe d'un retour à la "vérité ». Un accès plus facile aux archives permet aux chercheurs, parfois dans le cadre de projets internationaux, de traiter les documents avec de nouvelles approches et positions épistémologiques, donnant ainsi lieu à un renouveau historiographique.

3 Cet article s'intéresse particulièrement à la situation dans laquelle la question des alternatives au bolchévisme est alors soulevée. Signe d'une révision de l'histoire longtemps falsifiée, cette question anime aussi les débats politiques du moment qui exigent de faire un choix. Débattre autour des alternatives non réalisées en 1917 implique pour beaucoup de Soviétiques des réflexions plus générales sur leur histoire et sur le tournant que celle-ci doit prendre à la suite de la perestroïka. La publication par P. Volobouev (1987) de son livre sur le choix des voies de développement donne, dès 1987, une nouvelle orientation méthodologique aux historiens. Contrairement aux dogmes du 
Précis d'histoire du parti, ils posent des questions sur l'existence des autres issues pour les trois révolutions russes.

Ce débat ouvert par les historiens s'amplifie grâce à une nouvelle presse lancée par le Comité central cherchant à renforcer ses positions idéologiques fragilisées. La pratique des tables rondes réunissant spécialistes, représentants de l'appareil du parti et de l'intelligentsia est une autre façon de maîtriser l'histoire partant à la dérive sous les coups de la mémoire collective. C'est la revue Dialog, crée par le Comité central en 1990 et destinée à un public large qui diffuse l'entretien entre V. Melnitchenko, docteur en histoire et responsable du secteur idéologique du Comité central du PCUS et deux spécialistes de la révolution d'Octobre, l'académicien P. Volobouev, et le membrecorrespondant de l'Académie des sciences, J. Poliakov sous le titre Octobre 1917: le plus grand événement $d u X X^{e}$ siècle ou une catastrophe sociale? En 1991, la maison d'édition du parti Politizdat publie sous le même titre un recueil d'articles sous la direction de P. Volobouev. Pareille interrogation aurait été impensable quelques années auparavant, tant la révolution d'Octobre avait été l'objet de sacralisation depuis soixante-dix ans.

P. Volobouev réunit des articles parus dans la presse en 1989-1990. Parmi les auteurs russes (V. Bouldakov, A. Boutenko, G. Ioffé, V.Startsev, V. Loguinov et G. Sobolev) figurent des étrangers (J. Ellenstein, A. Rabinovitch, F. Moutchi) et deux historiens de la première émigration, P. Milioukov et S. Melgounov. L'épigraphe est de E. Carr. Concevoir ainsi un recueil est une façon de revendiquer le principe du pluralisme scientifique face au phénomène du remplacement hâtif et «incompétent » d'un monopole mythologique par un autre. Chacun des auteurs, conscient de la nécessité de réécrire l'histoire révolutionnaire, contribue à détruire le schéma stalinien et apporte ses réponses à tous ceux qui "par ignorance" se laissent guider par des ressentiments envers les révolutionnaires. La révolution d'Octobre s'en sort rétablie dans son statut d'un des plus grands événements du $\mathrm{xx}^{\mathrm{e}}$ siècle et l'interrogation sur la " catastrophe sociale » est levée grâce à l'inscription des révolutions russes dans le contexte mondial du début du $\mathrm{xx}^{\mathrm{e}}$ siècle.

6 Il ressort du recueil que des études historiques (marxistes comme non marxistes) montrent la révolution comme étant riche d'enseignements à l'heure de la perestroïka. En effet, savoir, au début des années 1990, que le processus historique n'est pas déterminé mais laisse un choix des possibles signifie un encouragement à l'action politique. De ce point de vue, une phrase timidement (ou prudemment?) entre parenthèses de P. Volobouev aurait dû sonner alors comme une révélation épistémologique : «Il est généralement admis et même incontestable que dans la situation actuelle de notre pays il n'y a pas d'alternative à la perestroïka. Or, cela ne signifie pas que dans la réalité il n'existe pas d'autres variantes de développement.» (Volobuev, 1991:141) L'analyse de l'article de P. Volobouev "Y avait-il une alternative? » ainsi que celle de l'ensemble du discours historiographique à ce sujet pointe là où la vie politique du pays fait mal : toutes les alternatives démocratiques apparaissent soit comme non réalisées, soit comme non réalisables. Bien qu'animé par le parti et les académiciens de son establishment et lancé à un moment fort opportun, l'intérêt à un jeu des possibles en 1917, semble remarquable par la pertinence du « pourquoi? ».

7 Chronologiquement, ce questionnement se concentre entre 1987 et 1994 quand il participe activement à la déconstruction de la vulgate marxiste-léniniste des formations socio-économiques ainsi que de la conception libérale et totalitaire de la révolution. Mais, historiquement, ce cadre est plus large et nécessite des explications sur l'avant et l'après 
épisode où l'intérêt pour une autre issue de la révolution russe eut des effets sur le réel des transformations initiées par Gorbatchev.

\section{Avant. Les révisionnistes}

Quelques premiers signes de réflexion sur la conception sacro-sacrée d'Octobre datent des années 1960-1970. Suite à la première déstalinisation, une nouvelle direction de recherches (novoe napravlenie) se profile alors avec une série de travaux consacrés au capitalisme en Russie (P.Volobouev, K. Tarnovski, I. Gindine, V. Danilov, A. Avrekh, A. Anfimov, P. Galouzo). Sans rompre explicitement avec la théorie de l'impérialisme de Lénine, ces auteurs se permettent d'intégrer dans leurs livres ou articles des documents qui interrogent sur le degré de développement du capitalisme avant la révolution. Alors, au lieu des industries monopolistes et des banques puissantes, apparaît une multitude d'ordres économiques (mnogoukladnost') parmi lesquels le capitalisme n'est pas dominant (Tarnovskij, 1972). Ce qui pose, certes implicitement, la question de l'alternative réformiste et incite à une nouvelle lecture de Lénine (novoe pročtenie). Elle tente en particulier M. Gefter au sujet des rapports entre le populisme et le léninisme vus jusquelà exclusivement sous l'angle de la rupture: les populistes petits bourgeois n'auraient rien en commun avec le leader du prolétariat (Gefter, 1969a). Gefter ose une lecture du jeune Lénine qui fait entrevoir une complexité (ou proximité) inattendue entre deux courants de pensée et deux pratiques révolutionnaires. À la tête d'un groupe de chercheurs (sektor) à l'Institut d'histoire universelle, il met à l'étude le problème des variantes en histoire (aujourd'hui, on dirait des alternatives) en ébranlant ainsi les certitudes du matérialisme historique (Gefter, 1969b).

9 À l'Institut d'histoire de Leningrad, un autre groupe d'historiens renouvelle la recherche sur la révolution d'Octobre. Le cadre conceptuel reste le même : Lénine a toujours raison dans les discussions avec ses adversaires, mais la description bien documentée de ces mêmes discussions fait connaître aux lecteurs les points de vue et les arguments des proscrits tels Zinoviev, Kamenev, Trotski (Startsev, 1965; Znamenski, 1964; Tokarev, 1967).

En outre, dans ces années, l'histoire des autres partis socialistes attire l'attention particulière des chercheurs. Ils les sortent de l'oubli bien qu'ils le fassent sous des titres conventionnels: L'effondrement du parti SR de gauche (Gusev, 1963); La banqueroute des partis petits-bourgeois en URSS (Gusev, 1966) ; L'effondrement des gouvernements SR (Garmiza, 1970); La chute des partis non prolétariens (Spirin, 1977).

11 Cependant, les réflexions hors norme que se permettent ces chercheurs sont vite désapprouvées. Les vigiles de la conception inébranlable y repèrent une hérésie : si le capitalisme n'était pas bien développé, la révolution n'aurait pas pu être socialiste ? Si on laisse entendre qu'il y avait de la spontanéité dans le déroulement de la révolution, on met en doute le rôle du parti et on suppose que du côté des autres partis socialistes il y avait des alternatives pour le développement de la société ? Inacceptable. Aux yeux des bureaucrates du parti, ces travaux passent pour «révisionnistes». Ce terme figure clairement dans une lettre adressée au Comité central par sept membres-zélateurs de l'Institut d'histoire universelle. Ce courant historiographique est réprimé en $1972^{1}$.

De l'autre côté du rideau de fer où règnent des conceptions conservatrice, libérale et totalitaire diamétralement opposées à la conception marxiste-léniniste, il y a une révision 
plus ouverte et plus approfondie. Elle se manifeste dans un contexte politique plus favorable à la recherche et aux échanges intellectuels. J'entends par là, la Détente, la crise du mouvement ouvrier et communiste à travers le monde, et un facteur plus important encore, le changement des paradigmes en sciences sociales : Bourdieu, Foucault, Barthes en Europe et le Linguistic Turn outre-Atlantique, qui initient la déconstruction du discours.

C'est dans ce contexte que le monde universitaire anglo-saxon réagit en premier quant au sujet des révolutions russes. C'est Leopold Haimson qui se distingue en tant que père fondateur d'un nouveau courant de pensée. Dans son article "The Problem of Social Stability in Urban Russia, 1905-1917 » (Slavic Review, 1964, nº 23 et 1965, n²4), il révise les prémisses de la révolution et arrive à la conclusion que les événements d'Octobre ne se réduisent pas à un coup d'État. La révolution est un phénomène social, non un enchaînement des hasards : la révolution de 1917 fut inévitable, tout comme ses issues imprévisibles. Il lance ainsi un défi à la conception libérale dominante et optimiste au sujet d'un réformisme possible, mais non réalisé. Il innove dans l'étude de la société comme autonome du pouvoir politique et des contraintes économiques et c'est tout un courant historiographique dit « révisionniste » qui le suit.

revisionnistes de la "première génération" s'attaquent à la théorie totalitaire appliquée à l'histoire soviétique. Les historiens qui traitent directement de la révolution ou des révolutionnaires (M. Fainsod, E. Carr, I. Deutscher, S. Cohen, A. Robinowitch) sont de ce fait plutôt novateurs en histoire politique.

C'est la deuxième génération de spécialistes guidée par S. Fitzpatrick qui, dans les années 1980, produit le plus sur l'histoire sociale de la révolution. Elle porte son attention sur les processus de radicalisation des masses et approfondit, par cette approche, l'analyse de la classe ouvrière, de la paysannerie et des prémisses de la révolution d'Octobre (S. Smith, A. N. Wildman, O. Figes) ${ }^{2}$.

En France où la conception libérale et totalitaire a beaucoup moins de prise sur le monde universitaire qu'en Amérique, c'est La révolution de 1917 de Marc Ferro parue en 1967-1976 qui propose une histoire révisée. Selon lui, l'insurrection d'Octobre ne se réduit pas au coup d'État bolchévique, car elle s'inscrit dans un mouvement révolutionnaire et populaire très large; elle n'est «ni fruit d'une nécessité historique, ni accident dû à la guerre et la défaite ». Il fait découvrir la spontanéité des masses et leur radicalisation, de février à octobre 1917, auparavant supposées sous le contrôle du parti bolchévique. En présentant les aspirations de la classe ouvrière, de la paysannerie, des soldats et des allogènes, Marc Ferro réoriente le regard, délaissant les grands personnages du parti bolchévique pour se tourner vers le comportement des hommes ordinaires. De plus, en utilisant les matériaux audiovisuels, il inaugure une toute nouvelle direction de recherche : le cinéma comme source de l'histoire.

Vers la fin des années 1980, les travaux des révisionnistes gagnent en prestige auprès des universités de plusieurs pays. Des historiens soviétiques eux-mêmes expriment leur intérêt pour certains de ces travaux qui renouvellent leur questionnement (Ioffe, Buldakov). On peut ainsi penser qu'avant la décomposition du camp socialiste et la désagrégation de l'URSS, la bipolarité conceptuelle des révolutions russes était prête à voler en éclat, à l'image du monde bipolaire. La fin de la guerre idéologique crée une atmosphère favorable à des recherches sans contraintes. 


\section{1987-1994. Le pic des débats}

Mais les recherches ne suivent pas un chemin tranquille. Vient la pérestroïka. Gorbatchev est le premier à semer la confusion dans la tête des Soviétiques, comme chez toute une armée de propagandistes du parti et d'historiens : il ne cesse de répéter que la pérestroïka est une suite de la révolution d'Octobre. Que veut-il dire par là ? Que le socialisme n'a pas été construit? Que les réformes signifient le retour à la NEP ? Qu'il y avait un jeu des possibles en 1917 resté insoupçonné?

erçu historiographique présenté ci-dessus ne concernait qu'un petit nombre d'historiens soviétiques tandis que la majorité restait enfermée dans le schématisme stalinien. À l'arrivée de la perestroïka, la situation change, les efforts de révision s'amplifient et cette fois, particulièrement autour des alternatives aux choix faits par les bolcheviks ${ }^{3}$.

Dans le domaine politique, c'est la question du caractère inévitable de l'insurrection armée et de la « dictature démocratique du prolétariat et de la paysannerie » en 1917 qui anime les débats. P. Volobouev, V. Bouldakov et plusieurs autres argumentent en faveur d'une possibilité réelle de résoudre la question du pouvoir sans insurrection et sans dictature : un accord entre les partis socialistes aurait pu réussir, ou un gouvernement des soviets aurait pu avoir sa chance (Volobuev \& Buldakov, 1996). Certains historiens voient les possibilités d'une issue démocratique en rapport avec l'Assemblée constituante ou la victoire des SR (Protasov \& Miller, 1993). D'autres, soucieux de comprendre les raisons de ce fiasco, passent au crible les erreurs du Gouvernement provisoire et des constitutionnels-démocrates (Starcev, 1989; Ioffe, 1995). L'histoire des partis démocratiques en 1917 semble alors offrir une moisson d'enseignements quant au changement de régime entamé sous Gorbatchev.

21 Dans le domaine économique, les possibilités réformistes d'avant 1917 sont moins discutées. C'est la NEP qui semble être une expérience à réétudier pour mieux gérer le passage à l'économie de marché prônée par les réformateurs de la perestroïka (Dmitrenko, 1994). Les historiens, ainsi que l'opinion publique, attribuent donc aux situations révolutionnaires et postrévolutionnaires des années 1920 le statut d'un point de départ raté qui vaudrait la peine d'être réexaminé à fond.

22 Un autre courant se disant " démocrate ", représenté moins par les historiens-spécialistes que par l'intelligentsia active depuis les années 1960, et de ce fait plus influent, se penche aussi sur le «pourquoi ?» mais en privilégiant plus nettement des approches inspirées par le changement des paradigmes en sciences sociales. J. Afanasiev, un des chefs de file de ce courant, appelle à aller jusqu'au bout dans la révision de l'ancienne conception de la révolution. Ce n'est qu'en la rejetant qu'on peut renouveler les questions à poser au passé. Pour lui, vue de la hauteur des sciences sociales de la fin du xxe siècle, l'idée du peuplevictime des tyrans, cultivée dans l'espace public, ne résiste pas aux critiques. Afanasiev est le premier à soulever publiquement la question de la responsabilité du peuple pour

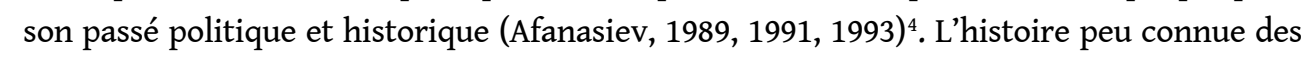
masses prises dans le tourbillon révolutionnaire attire l'attention des chercheurs sur les témoignages et les mémoires, éveillant ainsi l'intérêt du large public pour les péripéties des vies ordinaires. 
Pendant quelques années, la question de l'alternative au bolchévisme est vivement discutée dans la presse et autour de nombreuses tables rondes. Mais tout compte fait, il s'agit, dans une large mesure, d'une reprise des discussions menées dans les années 1920 et 1930 parmi les émigrés.

Pourquoi l'alternative réformiste a-t-elle été condamnée? Pourquoi les partis démocratiques ont-ils échoué face une dictature de type jacobine ? Pourquoi l'Assemblée constituante a-t-elle échoué? Y avait-t-il une possibilité d'un gouvernement démocratique après le 25 octobre ? À quel obstacle s'est heurtée l'alternative socialiste des mencheviks et des socialistes-révolutionnaires de gauche? À une radicalisation des masses comparable à celle qu'a connu la France, ou à la smuta (révolte insensée et impitoyable) à la russe ? La NEP était-elle une alternative thermidorienne ou socialiste ? Enfin, la révolution était-elle socialiste? Prolétarienne? Ou plutôt jacobine et prolétarienne?

Au cours de ces débats, la plupart des participants soviétiques accèdent et s'intéressent pour la première fois aux écrits des penseurs russes chassés par la révolution à l'étranger. Ils découvrent la parole des proscrits socio-démocrates mencheviks, des socialistesrévolutionnaires, des constitutionnels démocrates et de tant d'autres inconnus ou oubliés, grâce à la frénésie éditorialiste des maisons d'édition anciennes, comme nouvelles. L'héritage intellectuel de la première émigration acquiert alors une valeur surprenante pour la fin du Xx ${ }^{e}$ siècle. Certaines analyses des années 1920 et 1930 semblent clairvoyantes et incitent à les récupérer ou à les suivre. C'est le cas des smenovekhovtsy ${ }^{5}$ et des eurasistes. Par contre, la diffusion des connaissances des textes originaux écrits par les révolutionnaires de toute obédience, ainsi que le flot d'information ou de désinformation les concernant, détournent rapidement la société des alternatives socialistes et démocratiques. L'intérêt pour l'alternative boukharinienne cède vite la place à celui pour la sagesse d'État de Stolypine.

Après l'interdiction du parti et la désagrégation de l'URSS, un large public prête l'oreille à tous ceux qui mettent la tragédie révolutionnaire sur le compte des conspirateurs proanglais ou pro-allemands et par ricochet, les misères et les humiliations causées par la « thérapie de choc » de Egor Gajdar sur le compte des Européens malveillants.

\section{Après. La conception unique de la Grande révolution russe}

27 Quelle construction de la mémoire collective ressurgit alors de l'onde de choc de la perestroïka? Les efforts de réflexion collective (osmyslenie) sur l'échec du socialisme démocratique prôné à l'époque révolutionnaire s'épuisent. Et cela pour plusieurs raisons.

Discréditée, la poignée d'historiens animés par ce sujet n'est plus suivie, après l'interdiction du parti communiste qui les avait soutenus. Les ex-Soviétiques, effrayés par les révélations sur la terreur stalinienne, assimilent tous les malheurs postérieurs avec la révolution. La terreur rouge semble plus sanglante que celle perpétrée par les blancs. Les socialistes d'autrefois (mencheviks, SR, anarchistes) apparentés aux bolcheviks sont pris en haine. Sur le fond tragique de la guerre civile, le passé tsariste se dessine en rose.

$\mathrm{Au}$ bout de quelques années, l'intérêt réflexif de la population pour son passé faiblit. Les émotions nostalgiques pour "la Russie perdue ", tsariste, ou soviétique, ou les deux, agitent le corps collectif ${ }^{6}$. Un certain nombre d'historiens répondent aux attentes 
nostalgiques qui gagnent les Russes en reproduisant plus ou moins la polarisation classique autour des conceptions déjà rodées de la révolution. La conception libérale et totalitaire de R. Pipes, réaffirmée dans sa nouvelle édition, attire plus d'adeptes que les révisions savantes ou les réflexions des démocrates.

Enfin, une autre raison, celle qui se montre la plus forte, se fait sentir après «l'insurrection» d'octobre 1993 ou le "coup d'État d'Eltsine», comme on l'appelle maintenant. C'est, on peut dire, la raison d'État : j'entends par là une de ses premières manifestations, à savoir la Conférence du 5 novembre 1994 (Oktjabr' 1917, 1995).

C'est l'Administration du président Eltsine qui a pris l'initiative de réunir des historiens, des représentants de régions, et de différentes branches scientifiques, et même des hommes d'affaires, autrement dit un large public (obščestvennost'). Le discours de Sergueï Filatov, chef de l'Administration du président a donné le ton: selon sa définition, la révolution fut "l'événement le plus tragique de l'histoire millénaire de la Russie », « un coup d'État qui a permis à un petit groupuscule de la gauche radicale d'usurper le pouvoir », elle fut une affaire de « démons » à la Dostoïevski.

La révolution est à condamner, à oublier comme une catastrophe et une erreur, son expérience est à exclure des débats publics. Une page d'histoire à tourner. Cette conférence passe dans l'opinion des historiens pour celle qui donne les directives ( ustanovočnaja).

Depuis, la construction de la mémoire collective autour des révolutions russes et d'autres à travers le monde, y compris les « révolutions de couleur ", est entre les mains de ceux qui s'occupent de l'histoire publique. Il faut entendre par là une production massive de films historiques et documentaires, d'émissions télé, de romans historiques, de pièces de théâtre, de sites Internet, de revues et livres de vulgarisation, etc. Sans doute, cette production est-elle soutenue par des historiens professionnels institutionnellement bien établis qui trouvent l'approbation et le financement du gouvernement au détriment d'autres groupes d'historiens et de citoyens, qui tiennent à dépolitiser l'histoire mais qui ont du mal à se faire entendre.

Le président Poutine veut aussi, à sa façon, dépolitiser l'histoire. Face à la division de la société au sujet du centenaire de la révolution, il a fini, très tardivement, le 19 décembre 2016, par donner le feu vert aux préparatifs en suggérant de « respecter cet événement historique et de le traiter exclusivement comme tel, sans politiser le thème ». Pour exécuter son ordre, le 23 janvier 2017, un Comité d'organisation de la commémoration du centenaire a été constitué.

Quel dispositif le Comité a-t-il mis à l'œuvre ? Premièrement, « consolider la communauté des historiens autour d'une conception unique et objective de l'événement» (l'histoire n'est-elle pas un débat permanent?); deuxièmement, faire connaître cette conception auprès des universités, musées, bibliothèques et autres organismes culturels qui préparent des manifestations commémoratives dans le pays (n'est-ce pas une directive politisée ?).

Du point de vue épistémologique, le travail du Comité rappelle le Premier congrès des historiens-marxistes, en décembre 1928, dont la tâche consistait aussi à élaborer une seule et unique conception de la révolution de 1917, ainsi que de toute l'histoire mondiale. Sauf qu'à l'époque cette approche correspondait au positivisme ambiant, tandis qu'aujourd'hui elle ne tient compte ni de la reconnaissance a priori du jeu des possibles en 
histoire, ni du principe d'historicité, primordial pour l'historien comme pour la société, afin qu'elle puisse procéder au travail de mémoire et de réconciliation avec son passé.

Du point de vue conceptuel, c'est une construction qui ramène toute la complexité du phénomène révolutionnaire à la simplification pluriséculaire qui oppose la Russie à l'Occident. En effet, si on écoute le président du Comité d'organisation qui formule la conception unique, on l'entend dire que d'un côté, il y a les révolutions européennes, de l'autre - en contrepoids - la Grande révolution russe. Celle-ci " plonge ses racines dans la spécificité de la civilisation russe avec sa communauté rurale (obščina) et son aspiration à intégrer l'Europe en tant que membre égal tout en craignant pourtant une occidentalisation excessive». En qualifiant le marxisme "d'arme idéologique de l'occidentalisation", le président du Comité A. Torkounov, qui est aussi recteur de l'Institut des relations internationales, conclut comme l'avait déjà fait précédemment, en 2015, le ministre de la Culture V. Medinski : « La transformation révolutionnaire de la Russie a assuré la continuité entre l'Empire russe, l'URSS et la Fédération de Russie et a initié un projet global de civilisation à l'échelle mondiale ${ }^{7}$.»

\section{Pour conclure}

Au début de la déconstruction des conceptions bipolaires de la révolution de 1917, il y eut un court épisode promettant d'entamer un travail de mémoire collective dans la direction de la démocratisation du régime autoritaire. Or ce travail a été délaissé : le coup d'État d'Eltsine et le désastre économique des années 1990 lui ont coupé l'élan. Depuis, le présent postsoviétique n'a fait qu'accentuer le traumatisme de la révolution. On ne lui attribue qu'un seul sens positif: bien que cruelle, la politique de Staline, bon manager pragmatique, aurait permis de sauver la grandeur de l'empire tsariste en crise et de rendre évidente la supériorité de la civilisation russe. La conception unique, en fait, idéologique et géopolitique, élaborée par le Comité d'organisation ne fait que glisser sur un registre émotif de la mémoire collective une série de traumatismes : révolutions au pluriel, guerre civile, guerre patriotique, chaos des années 1990. Paradoxalement, la déconstruction d'une Grandeur aboutit à la construction d'une autre qui se démarque à nouveau par un isolationnisme agressif envers l'Occident.

\section{BIBLIOGRAPHIE}

AfANASIEV Jurij (1989), « Kto my takie? », Smena, 24 juin.

AFANASIEV Jurij (1991), « Ne stoit prazdnovat' datu, nad kotoroj nado razmyšljat' », Golos, 4-11 novembre.

AFANASIEV Jurij (1993), « Iz vseh variantov sud'by my vsegda vybiraem naihudšij », Golos, 15-23 mars.

DMITRENKo V. P. (1994), NÈP. Priobretenija i poteri, Moscou : Nauka. 
GEFTER Mihail Ja. (1969a), « Iz istorii leninskoj mysli », Novyj Mir, 4 ; une version incomplète en français : M. Gefter (1970), « Lénine et la perspective historique », Recherches internationales à la lumière du marxisme, 62.

GEFTER Mihail Ja. (1969b), Istoričeskaja nauka i nekotorye problemy sovremennosti, Moscou.

GUSEV Kirill V. (1963), Krah partii levyh èserov, Moscou.

GUSEV Kirill V. (1966), Krah melko-buržuaznyh partij v SSSR, Moscou.

IofFE Genrih Z. (1995), Semnadcatyj god: Lenin, Kerenskij, Kornilov, Moscou.

Oktjabr' 1917 i bol'ševistskij èksperiment v Rossii (1994), Materialy konferencii 5 nojabrja 1994.

PRotasov L. G. \& MilleR G. I. (1993), « Vserossijskoe učreditel'noe sobraznie i demokratičeskaja al'ternativa; Dva vzgljada na problemu », Otečestvennaja istorija, 5.

SPIRIN Leonid M. (1977), Krušenie pomeščičih i buržuaznyh partij v Rossii (nac. XX-1920), Moscou.

STARCEV V. I. (1965), Očerki po istorii Petrogradskoj Krasnoj gvardii i rabočej milicii, Moscou-

Leningrad : Nauka.

TARNOVSKIJ K. (dir.) (1972), Voprosy istorii kapitalističeskoj Rossii. Problema mnogoukladnosti, Sverdlovsk.

TOKAREv Ju. S. (1967), Aprel'skij krizis, Leningrad.

VolobueV Pavel (1987), Vybor putej obščestvennogo razvitija: teoria, istorija, sovremennost', Moscou : Politizdat.

Volobuev Pavel (dir.) (1991), Oktjabr'skaja revoljucija: glavnoe sobytie XX veka ili tragičeskaja ošibka?, Moscou : Politizdat.

VolobUev Pavel (1995), « Istoričeskie korni oktjabr'skoj revoljucii », Anatomija revoljucii, SaintPétersbourg.

VOLOBUEV Pavel \& BULDAKOV Vladimir (1996), « Oktjabr'skaja revoljucija: novye podhody k

izučeniju », Voprosy istorii, 5-6.

ZNAMENSKIJ Oleg Nikolaevič (1964), Ijul'skij krizis, Leningrad.

\section{NOTES}

1. Voir les attaques des historiens officiels: «Obsuždenie nekotoryh problem metodologii istorii », Voprosy istorii, 1971, $\mathrm{n}^{\circ} 10$; Neretina S., « Istorija s metodologiej ili konec istorii », Vek XX i Mir, 1996, $\mathrm{n}^{\circ} 1$.

2. Pour l'évolution de l'historiographie anglophone, voir : Ėkton E்., « Novyj vzgljad na russkuju revoljuciju », Otečestvennaja istorija, 1997, nº 5 ; Ševyrin V. M. (dir.), 1917 god. Rossija revoljucionnaja, Moscou : INION, 2009.

3. Vu le nombre élevé de publications à ce sujet, mentionnons trois références qui saisissent l'essentiel des débats : Rossija 1917 god: vybor istoričeskogo puti, Moscou : Nauka, 1989 ; Oktjabr'skaja revoljucija. Narod: ee tvorec ili založnik, Moscou, 1992 ; et une thèse soutenue en 2001 à l'université de Tjumen': Pridorožnyj A.V., Problema al'ternativ obščestvennogo razvitija perioda revoljucii 1917 goda $v$ otečestvennoj istoriogrfii.

4. Marc Ferro a déjà abordé cette question en 1980 dans son ouvrage Des soviets au communisme bureaucratique : les mécanismes d'une subversion. 
5. Le « Changement de jalons » (Smena Vekhov) est un recueil d'articles publiés à Prague en 1921 par des émigrés russes.

6. Un chercheur tenté par l'histoire des émotions en vogue y trouvera son miel.

7. Documents en ligne: le discours de V.Medinski, «Kruglyj stol 100 let Velikoj rossijskoj revoljucii: osmyslenie vo imja consolidacii » (20 mai 2015) sur pravmir.ru ou YouTube vidéo; le message de V. Poutine, "100-letie Oktjabr'skoj revoljucii otmetjat vne politiki » (28 décembre 2016) sur postsovet.ru ; le texte de l'ordre du Président (20 décembre 2016) sur rushistory.org ; le discours de A. Torkunov, "Pervoe zasedanie orgkomiteta, posvjaščennoe 100-letiju Revoljucii 1917 goda » (24 janvier 2017) sur rushistory.org.

\section{RÉSUMÉS}

Suite à la perestroïka, un temps (1987-1994) de débats publics au sujet des possibilités démocratiques ratées de 1917. Un temps aussi pour comprendre les changements politiques en cours et se projeter vers un avenir démocratique réussi. À l'heure du centenaire, il s'agit toujours d'un travail de mémoire non accompli.

Following the perestroika, a time (1987-1994) of public debates about the failed democratic possibilities of 1917. A time also to understand the political changes in progress and to plan for a successful democratic future. At the time of the centenary, it is always an unfinished work of memory.

\section{INDEX}

Mots-clés : perestroïka, débats publics, alternatives démocratiques, centenaire de 1917, travail de mémoire

Keywords : perestroika, public debates, democratic alternatives, centenary of 1917, memory work

\section{AUTEUR}

\section{TAMARA KONDRATIEVA}

Tamara Kondratieva, a fait ses études et soutenu sa thèse à l'université de Moscou, son doctorat et l'HDR à Paris 1 Panthéon-Sorbonne. Elle a enseigné à l'INALCO et à l'université de Valenciennes et du Hainaut-Cambrésis. Depuis 2014, professeur émérite en histoire contemporaine, elle est l'auteur de : La Russie ancienne, Paris, PUF, coll. « Que sais-je ?», 1996 ; Bolcheviks et Jacobins. Itinéraire des analogies, Paris, Payot, 1989, $2^{\mathrm{e}}$ éd. Les Belles lettres, 2017 ; Gouverner et nourrir. Du pouvoir en Russie (Xvi ${ }^{e}-\mathrm{XX}^{e}$ siècles), Paris, Les Belles Lettres, 2002 ; (dir.) Les Soviétiques, un pouvoir, des régimes, Paris, Les Belles Lettres, 2011. 